\title{
Fulminant Emphysematous Hepatitis - A Rare Cause of Septic Shock
}

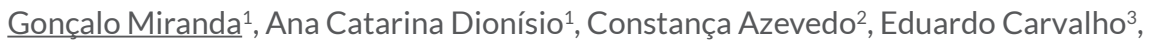 \\ Miguel Semião ${ }^{2,4}$, Vítor Branco ${ }^{3,4}$, Miguel Castelo-Branco ${ }^{3,4}$ \\ ${ }^{1}$ Internal Medicine Department, Centro Hospitalar Universitário Cova da Beira, Covilha, Portugal \\ ${ }^{2}$ Surgery Department, Centro Hospitalar Universitário Cova da Beira, Covilha, Portugal \\ ${ }^{3}$ Intensive Care Medicine Department, Centro Hospitalar Universitário Cova da Beira, Covilha, Portugal \\ ${ }^{4}$ Faculdade de Ciências da Saúde, Universidade da Beira Interior, Covilha, Portugal
}

Received: 31/01/2020

Accepted: 03/02/2020

Published: $25 / 03 / 2020$

\begin{abstract}
How to cite this article: Miranda G, Dionísio AC, Azevedo C, Carvalho E, Semião M, Branco V, Castelo-Branco M. Fulminant emphysematous hepatitis - a
\end{abstract} rare cause of septic shock. EJCRIM 2020;7: doi:10.12890/2020_001539.

Conflicts of Interests: The Authors declare that there are no competing interests.

Acknowledgements: Gonçalo Miranda, Ana Catarina Dionísio and Constança Azevedo contributed equally to this work as first authors.

This article is licensed under a Commons Attribution Non-Commercial 4.0 License

\section{ABSTRACT}

Emphysematous hepatitis is a rare entity characterized by the replacement of hepatic parenchyma by gas, leading to acute liver failure. Often it occurs in patients with diabetes mellitus, liver disease or a recent history of abdominal surgery. We present a case of emphysematous hepatitis in a 74-year-old man with no recognizable risk factors. Despite the early broad-spectrum antimicrobial therapy and supportive care, the condition progressed to a fatal outcome, as seen in other case reports. Early recognition of this condition and rapid and aggressive management may improve patient outcomes.

\section{LEARNING POINTS}

- Emphysematous hepatitis is a rare condition characterized by replacement of hepatic parenchyma by gas.

- The diagnosis of emphysematous hepatitis requires imaging, preferably a computed tomography scan.

- Emphysematous hepatitis warrants awareness among clinicians for early diagnosis and rapid and aggressive management.

\section{KEYWORDS}

Fulminant emphysematous hepatitis, acute liver failure, septic shock

\section{CASE PRESENTATION}

A 74-year-old man was admitted to the emergency department (ED) of our hospital with altered mental status. He complained of fever, cough with mucopurulent sputum, and abdominal pain for 2 days. He had a medical history of hypertension, gastroesophageal reflux, and moderate alcohol consumption. His usual medication was lansoprazole $20 \mathrm{mg}$ daily, perindopril $5 \mathrm{mg}$ daily, and amlodipine $5 \mathrm{mg}$ daily, with no known medical allergies. On examination, he was disoriented and uncooperative. His vital signs included blood pressure of $135 / 85 \mathrm{mmHg}$, heart rate of 103 beats/min, respiratory frequency of 18 breaths/min, peripheral oxygen saturation of 85\% on room air, and tympanic temperature of $39.6{ }^{\circ} \mathrm{C}$. His skin showed signs of livedo reticularis and his capillary refill time was increased. Pulmonary auscultation showed bilateral rhonchi. On abdominal palpation, he complained of intense epigastric and right hypochondrium pain with muscle guarding. Laboratory examination revealed hypoxic respiratory failure, leukocytosis with neutrophilia, and a significantly altered liver profile: aspartate aminotransferase $1896 \mathrm{U} / \mathrm{L}$; alanine aminotransferase $640 \mathrm{U} / \mathrm{L}$; lactate dehydrogenase $2499 \mathrm{U} / \mathrm{L}$; alkaline phosphatase $230 \mathrm{U} / \mathrm{L}$; total bilirubin $4.78 \mathrm{mg} / \mathrm{dL}$. 
During his stay in ED, his blood lactate level increased from 5.1 to $10.2 \mathrm{mmol} / \mathrm{L}$ and C-reactive protein from 1.35 to $12.19 \mathrm{mg} / \mathrm{dL}$. Procalcitonin was $83.86 \mathrm{ng} / \mathrm{mL}$. Blood cultures were sent to the laboratory and empiric broad-spectrum antibiotics (piperacillin/tazobactam) were started. Assuming a possible abdominal focus, we performed an abdominal computed tomography (CT) scan with contrast. It revealed a diffuse heterogeneous structure with hypodensity within the right lobe of the liver and two relatively extensive areas with gas, the largest of which was $93 \mathrm{~mm}$ in diameter. There was no evidence of abscess formation or other significant changes (Figs 1 and 2). This result, along with the liver profile alterations, led to the diagnosis of emphysematous hepatitis with acute liver failure. While still in ED, the patient developed severe metabolic acidosis and distributive shock requiring vasopressor support. Because of his condition and the lack of proven benefit of a surgical approach, including percutaneous drainage, he was admitted to the intensive care unit and supportive therapy was instituted.

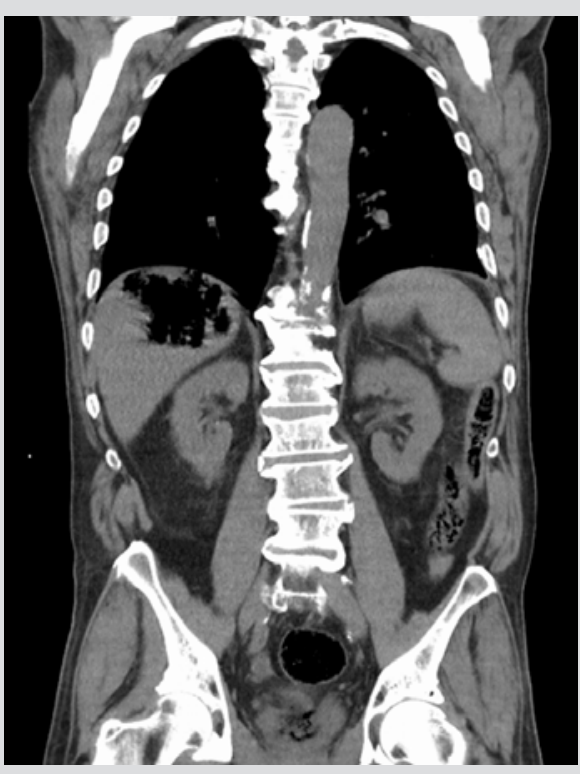

Figure 1. CT scan of the abdomen in coronal view showing an extensive area with gas within the right lobe of the liver

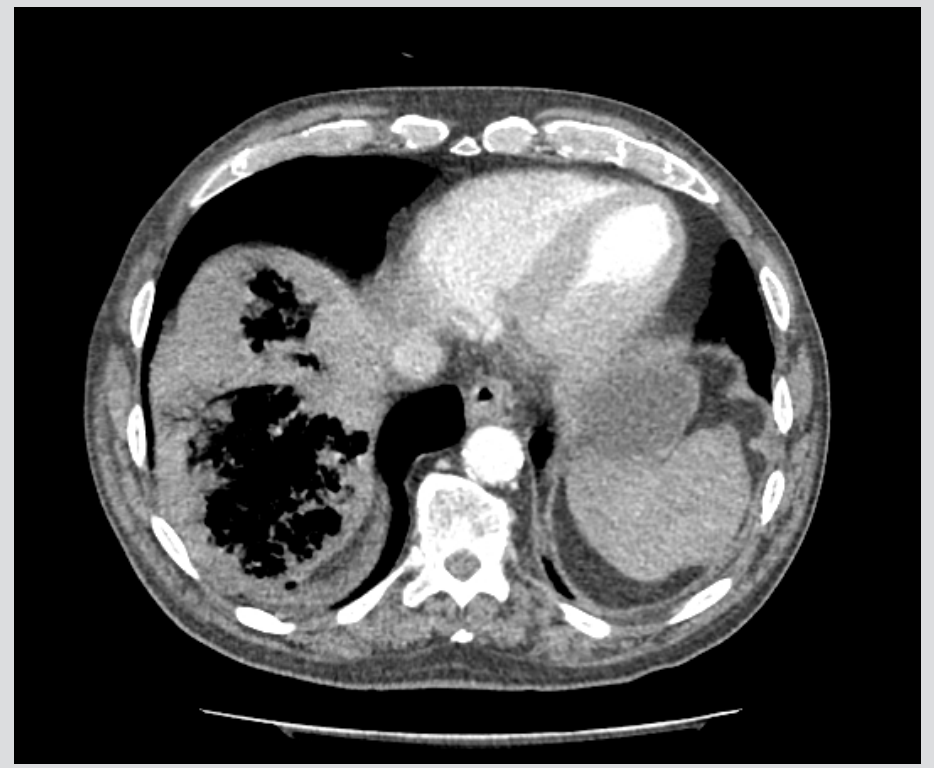

Figure 2. CT scan of the abdomen with contrast in axial view showing two areas with gas within the right lobe of the liver

On the second day of admission, his clinical state deteriorated along with blood analysis; the antimicrobial spectrum was extended and he was commenced on vancomycin, metronidazole, and fluconazole. Despite these measures, his condition evolved to multiorgan failure and he died on the third day of admission. The decision for autopsy was deterred due to lack of consent. Blood cultures subsequently grew multisensitive Escherichia coli.

\section{DISCUSSION}

Emphysematous infections of the abdominal organs have long been recognized and are potentially life-threatening conditions. The presence of gas within the liver can be due to a variety of causes, often including liver abscesses, iatrogenic causes, and seldom infection by gasforming bacteria ${ }^{[1]}$.

Emphysematous hepatitis is a rare condition characterized by replacement of hepatic parenchyma by gas often followed by a fulminant inflammation of the liver leading to acute liver failure, as seen in patients with risk factors such as diabetes mellitus and liver disease ${ }^{[1-4]}$ or a recent history of abdominal surgery ${ }^{[2-5]}$. In contrast to the other cases reported, our patient had no recognizable risk factors. Gas formation might develop in the setting of gas-forming infection, high tissue glucose level, and impaired transportation of gas by vascular compromise. Mixed acid fermentation from tissue necrosis by bacteria produce nitrogen, hydrogen, carbon dioxide, and oxygen, resulting in

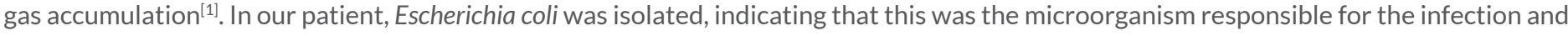
the emphysematous infiltration of the liver. Clinical manifestations are usually subtle at the beginning and progress rapidly, as seen in other reported cases ${ }^{[1-5]}$. Our patient complained of fever and abdominal pain for 2 days and was admitted with altered mental status, rapidly progressing to septic shock. The diagnosis of emphysematous hepatitis requires abdominal imaging, preferably a contrast-enhanced CT 
scan. Gas within the liver is usually evident but pyogenic liver abscesses should be ruled out.

Management of this condition usually involves parenteral broad-spectrum antibiotics and drainage of the collection. In all reported cases early broad-spectrum antibiotics were commenced but drainage was not always attempted either because it seemed futile ${ }^{[3]}$ or the hemodynamic status of the patient did not permit it ${ }^{[4]}$. In our patient, drainage was not attempted because it seemed futile and the patient's condition deteriorated so rapidly that the procedure could not be safely performed.

Emphysematous hepatitis continues to be difficult to manage and despite aggressive measures the condition progresses to a fatal outcome in the first days of admission, as seen in our case and other reported cases. We encourage the use of aggressive early therapeutic measures in patients with emphysematous hepatitis in order to control the infection.

\section{REFERENCES}

1. Chauhan U, Prabhu SM, Shetty GS, Solanki RS, Udiya AK, Singh A. Emphysematous hepatitis - a fatal infection in diabetic patients: case report. Clin Res Hepatol Gastroenterol 2012;36(6):e114-e116.

2. Blachar A, Federle MP, Brancatelli G. Acute fulminant hepatic infection causing fatal 'emphysematous hepatitis': case report. Abdom Imaging 2002;27(2):188-190.

3. Létourneau-Guillon L, Audet P, Plasse M, Lepanto L. Answer to case of the month \#162. Emphysematous infection of the liver parenchyma. Can Assoc Radiol J 2010;61(2):117119.

4. Nada KM, El Husseini I, Abu Hishmeh ME, Shah NS, Ibragimova N, Basir R. A rare case of septic shock secondary to emphysematous hepatitis. Case Rep Crit Care 2017;2017:1-3.

5. Kim JH, Jung ES, Jeong SH, Kim JS, Ku YS, Hahm KB, et al. A case of emphysematous hepatitis with spontaneous pneumoperitoneum in a patient with hilar cholangiocarcinoma. Korean J Hepatol 2012;18(1):94-97. 